\title{
The Deflation Accelerated Schwarz Method for CFD
}

\author{
J. Verkaik ${ }^{1}$, C. Vuik ${ }^{2, \star}$, B.D. Paarhuis ${ }^{1}$, and A. Twerda ${ }^{1}$ \\ 1 TNO Science and Industry, Stieltjesweg 1, P.O. Box 155, 2600 AD Delft, \\ The Netherlands \\ 2 Delft University of Technology, Faculty of Electrical Engineering, Mathematics and \\ Computer Science, Mekelweg 4, 2628 CK Delft, The Netherlands \\ Phone: +31 1527 85530, Fax: +31 152787209 \\ c.vuik@ewi.tudelft.nl.
}

\begin{abstract}
Accurate simulation of glass melting furnaces requires the solution of very large linear algebraic systems of equations. To solve these equations efficiently a Schwarz domain decomposition (multi-block) method can be used. However, it can be observed that the convergence of the Schwarz method deteriorates when a large number of subdomains is used. This is due to small eigenvalues arising from the domain decomposition which slow down the convergence. Recently, a deflation approach was proposed to solve this problem using constant approximate eigenvectors. This paper generalizes this view to piecewise linear vectors and results for two CFD problems are presented. It can be observed that the number of iterations and wall clock time decrease considerably. The reason for this is that the norm of the initial residual is much smaller and the rate of convergence is higher.
\end{abstract}

Keywords: efficiency; computational fluid dynamics; domain decomposition; deflation; Krylov subspace acceleration.

\section{Introduction}

Simulation by Computational Fluid Dynamics (CFD) is important for the design, optimization and trouble shooting of glass melting furnaces. It gives engineers in the glass industry great insight into the occurring transport phenomena. At TNO Science and Industry, a CFD simulation package called GTM-X is being developed for simulating gas- and oil-fired glass-furnaces. This is a complete model for simulating glass furnaces, describing the glass melt and combustion space simultaneously, and predicting the effects on melting performance and glass quality.

A domain decomposition (DD) approach is applied within GTM-X, for which the spatial domain is decomposed into subdomains (blocks). A DD (or multiblock) approach has several important advantages for simulating glass-melting

\footnotetext{
* Corresponding author.
} 
furnaces. Since a glass-furnace geometry is often complex, this enables us to easily describe the geometry using blocks (subdomains). Furthermore, parallel computing can be done and variables for each block can be simultaneously computed on different processors. This is an advantage, because simulation of glass-melting furnaces often results in very large computation times.

A disadvantage of multi-block solvers is that the convergence behavior deteriorates significantly when a large number of blocks is used. This is especially the case for solving elliptic equations. In [11, a reason for this problem is given which relates the loss of convergence to the presence of small eigenvalues arising from the domain decomposition. These eigenvalues can be 'eliminated' by applying a deflation operator using constant vectors for approximating the corresponding eigenvectors. The authors of [11] present convergence rates for solving Poisson's equation which are independent on the number of blocks.

In this paper we extend and apply this idea to solve general linear systems of equations. The deflation method is implemented in GTM-X and both GCR and CG Krylov subspace acceleration is used to solve the resulting linear system of equations. Both constant (CD) and combined constant and linear deflation (CLD) vectors are considered. The research in this paper focusses on solving the singular pressure-correction system in the SIMPLE method, which is used in GTM-X to solve the Navier-Stokes equations. However, the solver can be used for general linear systems and is therefore applicable to a wide range of problems.

\section{Description of the Mathematical Model}

Besides the incompressible Navier-Stokes equations and the energy equation, GTM-X has dedicated models for turbulence, combustion, glass melting and chemical vaporization. The user can apply different models in different domains.

The equations arising from the physical models are discretised with the finite volume method. Several schemes can be used for discretization: upwind, central, TVD higher-order schemes, and blending of schemes can be done using deferred correction. The grid is boundary fitting and colocated, meaning that all variables are located in the volume cell centers. The discretised nonlinear Navier-Stokes equations are solved by the SIMPLE method ([6, 1, 12, ), using pressure-weighted (Rhie \& Chow) interpolation to exclude checkerboard pressure modes. The SIMPLE method is an iterative method in which the system in each iteration (outer iteration) is splitted up into linear systems for the pseudo-velocities and pressure-correction. So-called SIMPLE Stabilization Iterations (SSI) are applied, which can be seen as additional outer iterations for solving the pressure system without solving the velocities. Linear systems of equations are solved with a domain multi-block approach (inner iteration). In GTM-X, an additive Schwarz DD method with minimal overlap is applied, in combination with inaccurate subdomain solutions. Stone's SIP-solver [7], which is based on an incomplete LU decomposition, is used for obtaining the subdomain 
solutions. Local grid refinement at block level can be done, as well as parallel computing using MPI libraries. Furthermore, solutions on coarse grids can be used as starting solutions for finer grids by using interpolation. The reader is referred to [10] for more details on the code.

\section{Deflation and Domain Decomposition}

Consider a decomposition of the entire computational domain consisting of $n$ grid points into $L$ nonoverlapping subdomains. Discretization of the partial differential equations to be solved and grouping together the unknowns per subdomain, results in the block system

$$
\left[\begin{array}{ccc}
A_{11} & \cdots & A_{1 L} \\
\vdots & \ddots & \vdots \\
A_{L 1} & \cdots & A_{L L}
\end{array}\right]\left[\begin{array}{c}
\boldsymbol{y}_{1} \\
\vdots \\
\boldsymbol{y}_{L}
\end{array}\right]=\left[\begin{array}{c}
\boldsymbol{b}_{1} \\
\vdots \\
\boldsymbol{b}_{L}
\end{array}\right]
$$

which we will denote simply as $A \boldsymbol{y}=\boldsymbol{b}$. The diagonal blocks of $A$ are the coefficients for the interior of the subdomains, the off-diagonal blocks represent coupling across subdomain boundaries. This system is solved with the deflation method combined with a Krylov subspace method.

Let the matrix $Z$ be $n \times m$, where $m \leq n$. Furthermore, let $Z$ be of rank $m$, i.e. $Z$ has linear independent columns. The columns of $Z$, the so-called deflation vectors, span the deflation subspace, i.e., the space that approximates the eigenspace belonging to the smallest eigenvalues and which is to be projected out of the residual. To do this we define the projectors

$$
P=I-A Z E^{-1} Z^{T}, \quad Q=I-Z E^{-1} Z^{T} A,
$$

where the $m \times m$ matrix $E$ is defined as $E=Z^{T} A Z$, and $I$ is the identity matrix of appropriate size. To solve $A \boldsymbol{y}=\boldsymbol{b}$ with the deflation technique, we write $\boldsymbol{y}=(I-Q) \boldsymbol{y}+Q \boldsymbol{y}$, and since

$$
(I-Q) \boldsymbol{y}=Z\left(Z^{T} A Z\right)^{-1} Z^{T} A \boldsymbol{y}=Z E^{-1} Z^{T} \boldsymbol{b},
$$

can be computed immediately, we only need to compute $Q \boldsymbol{y}$. In light of the identity $A Q=P A$ we can solve the system

$$
P A \tilde{\boldsymbol{y}}=P \boldsymbol{b}
$$

for $\tilde{\boldsymbol{y}}$, premultiply this by $Q$ and add it to (1).

Since $P A Z=0$, this system is obviously singular, and therefore no unique solution exists. However, it can be shown that $Q \boldsymbol{y}$ is unique [9]. In [2] and [8-p. 147], it is noted that a positive semidefinite system can be solved as long as it is consistent, i.e., as long as its right-hand side does not have components in the null space (column space of $Z$ ) of $P A$. This assumption holds for (2), because 


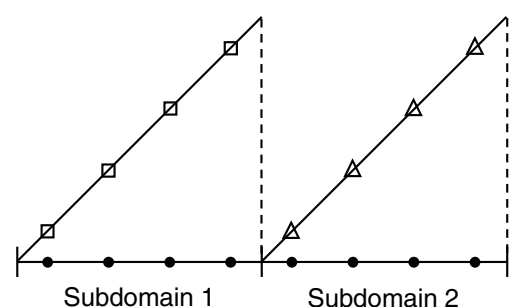

Fig. 1. Linear deflation vectors for the 1-D example of two subdomains with 4 grid cells per subdomain

the same projection is applied to both sides of $A \boldsymbol{y}=\boldsymbol{b}$. In [8-p. 147], it is shown that a Krylov subspace method could be used to solve (2), because the null-space never enters the iteration, and therefore the corresponding zero-eigenvalues do not influence the convergence. The reader is referred to 4] for more details on the spectrum of $P A$.

When system (2) is solved, this involves the computation of $E^{-1}$, for example statements like $\boldsymbol{f}=E^{-1} \boldsymbol{g}$ have to be evaluated. In [4-Section 3], it is shown that deflation works correctly as long as $E^{-1}$ is computed with a high accuracy. Since we never determine $E^{-1}$ explicitly, this means that $E \boldsymbol{f}=\boldsymbol{g}$ is solved by a direct method, for example by an $L U$ factorization.

Deflation can also be combined with preconditioning. Suppose $K$ is a suitable preconditioner of $A$, then solving $A \boldsymbol{y}=\boldsymbol{b}$ can be replaced by: solve $\tilde{\boldsymbol{y}}$ from the left-preconditioned system $K^{-1} P A \tilde{\boldsymbol{y}}=K^{-1} P \boldsymbol{b}$, and form $Q \tilde{\boldsymbol{y}}$, or solve $\tilde{\boldsymbol{y}}$ from the right-preconditioned system $P A K^{-1} \tilde{\boldsymbol{y}}=P \boldsymbol{b}$, and form $Q K^{-1} \tilde{\boldsymbol{y}}$. Both systems can be solved by a one's favorite Krylov subspace solver, for example by the GCR method. In our implementation we take $K$ the block Gauss-Jacobi preconditioner (block diagonal of $A$ ), corresponding to the additive Schwarz iteration. This preconditioner lends itself well for parallel computing. We will refer to the deflation method combined with Krylov subspace acceleration in a domain decomposition context as the deflated Krylov-Schwarz method.

\section{Approximating Eigenvectors by Constant and Linear Functions}

Many authors [3, 5, 11] consider constant deflation vectors for approximating the eigenvectors corresponding to the small eigenvalues which slow down the convergence. For each subdomain, exactly one deflation vector is defined having elements that are constant in the grid points on the corresponding subdomain, and zero elements in the grid points on the other subdomains. We will refer to this as CD deflation. Generalization to the 2-D and 3-D case is straightforward: for a subdomain, we simply take the elements of the deflation vector to be constant in the grid nodes. 
This idea is generalized to approximating the eigenvectors using piecewise linear vectors in the grid directions 1 . By this we augment the space of constant vectors with linear vectors. Figure 1 shows the linear deflation vectors. A linear deflation vector is defined as a polynomial of degree one on each subdomain and zero on the other subdomains. If the deflation vectors consist of both the constant and the linear vectors, we will denote it by Constant Linear Deflation (CLD). For the case of Figure 1], we have two deflation vectors per subdomain: one constant and one linear. Generalization to 2-D and 3-D is not so straightforward anymore, compared to CD deflation. However, it appears that for the 2-D case three vectors are connected to each subdomain: one constant, and one linear vector in each of the two grid directions. For the 3-D case, it appears that we need four deflation vectors: one constant and one linear vector in each of the three grid directions.

When the coefficient matrix $A$ is singular then the deflation vectors has to be chosen such that $Z^{T} A Z$ is non-singular, since break-down can occur with a direct solution method. Two options seem satisfactory to overcome this problem. The first one is to remove a constant deflation vector for one subdomain; the second is to adjust one entry in a constant deflation vector for one subdomain.

\section{$5 \quad$ Numerical Experiments}

The deflated GCR method is compared to the SIP method for solving the singular pressure-correction system arising in the SIMPLE method applied to the stationary incompressible Navier-Stokes equations. Two test cases are considered: a buoyancy-driven cavity flow for a high Rayleigh number, and a glass tank. In both test cases the stationary energy equation is solved besides the Navier-Stokes equations. Furthermore, the flow is assumed to be laminar and buoyant. One constant deflation vector is removed in order to overcome the singularity. Furthermore, one SIP iteration is used for obtaining the subdomain solution and the GCR algorithm is truncated for one search direction. In this paper we will restrict ourselves to the results for the inner iterations.

The first test case is a 2-D buoyancy-driven cavity flow in a unit square consisting of a $60 \times 60$ uniform grid and a decomposition of $4 \times 4$ subdomains, see Figure 2. The Rayleigh number is chosen to be large, i.e. $\mathrm{Ra}=1.0 \cdot 10^{6}$, meaning that the flow is highly buoyant but still laminar. Figure 3 shows the residuals for solving the pressure-correction system. Clearly, the CLD deflation performs best, followed by CD deflation and the GCR without deflation. The SIP method performs very disappointing for this case. Note that a large jump in the initial residual can be observed for CLD deflation.

\footnotetext{
${ }^{1}$ In this paper we let deflation vectors in the coordinate directions out of consideration. However, numerical experiments show that choosing linear vectors in grid- or coordinate directions can result in different convergence behavior.
} 


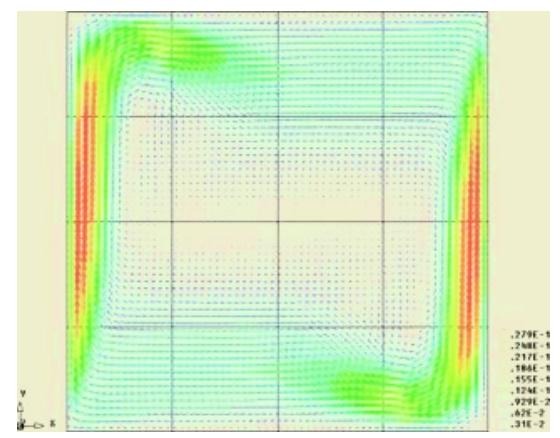

Fig. 2. Velocity field for the buoyancy-driven cavity flow

In the second test case, a glass tank is considered having dimensions $7[\mathrm{~m}]$ $\times 3[\mathrm{~m}] \times 1[\mathrm{~m}]$, see Figure 4. Glass fractions are injected (left of figure) from above and melted glass with desired properties leaves the tank at the outlet (right of figure). For this case the grid is uniform consisting of 10,500 grid cells, and the domain is decomposed into 18 subdomains. Figure 5 shows the results for the inner iteration residuals. Clearly, CLD deflation performs best concerning convergence behavior.

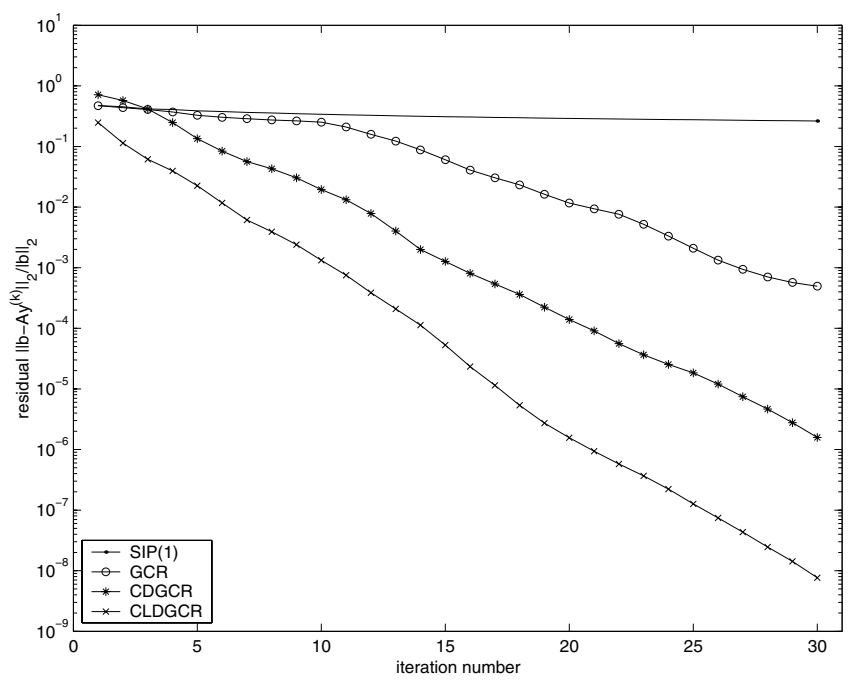

Fig. 3. The inner iteration residuals for the SIP and deflated GCR method considering the buoyancy-driven test case (GCR: GCR without deflation; CDGCR: GCR with CD deflation; CLDGCR: GCR with CLD deflation) 


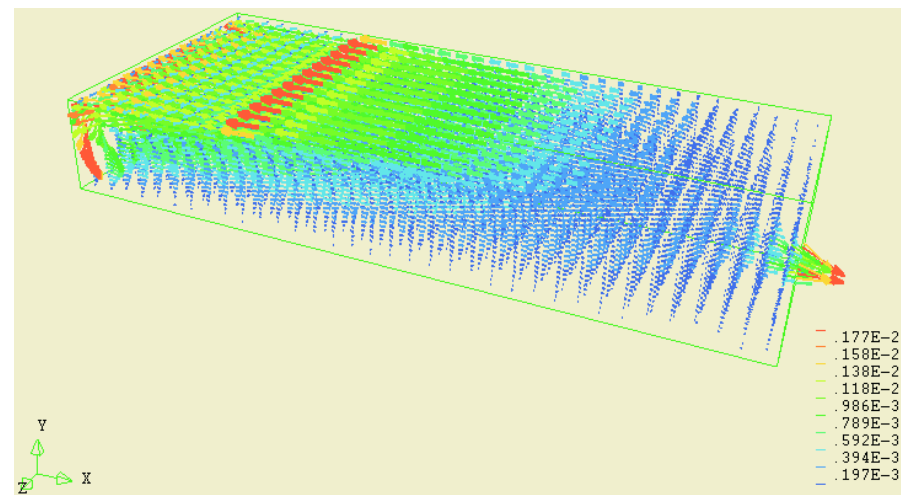

Fig. 4. Velocity field for the glass tank test case

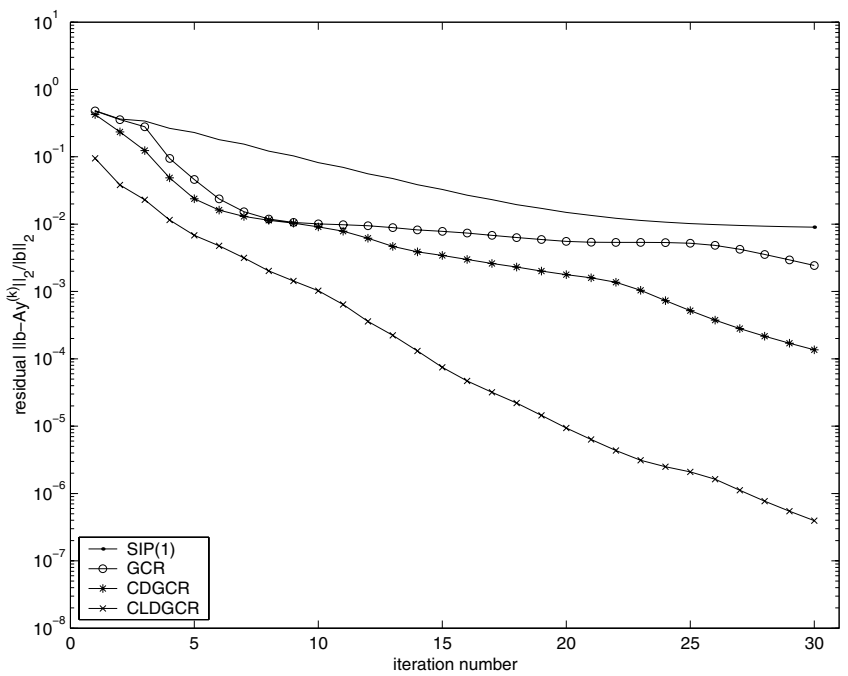

Fig. 5. The residuals of the inner iterations for the SIP and deflated GCR method considering the glas tank test case (GCR: GCR without deflation; CDGCR: GCR with CD deflation; CLDGCR: GCR with CLD deflation)

\section{Conclusions}

From the experiments presented in this paper, we conclude that the deflation accelerated Schwarz method is a very efficient technique to solve linear systems of equations arising from domain decomposition. It seems that the combination of constant and linear deflation vectors is most efficient. A large jump in the norm of the initial residual can be observed as well as a higher convergence rate. Moreover, it should be noted that the deflation method can be implemented in existing software with relatively low effort. 


\section{References}

1. J.H. Ferziger and M. Perić. Computational Methods for Fluid Dynamics. Springer, Heidelberg, second edition, 1999.

2. E.F. Kaasschieter. Preconditioned conjugate gradients for solving singular systems. J. Comp. Applied Math., 41:265-275, 1988.

3. L. Mansfield. On the conjugate gradient solution of the Schur complement system obtained from domain decomposition. SIAM J. Numer. Anal., 7(6):1612-1620, 1990.

4. R. Nabben and C. Vuik. A comparison of deflation and coarse grid correction applied to porous media flow. Report 03-10, Delft University of Technology, Department of Applied Mathematical Analysis, Delft, 2003.

5. R.A. Nicolaides. Deflation of conjugate gradients with applications to boundary value problems. SIAM J. Numer. Anal., 24(2):355-365, 1987.

6. S.V. Patankar. Numerical Heat Transfer and Fluid Flow. McGraw-Hill, New York, 1980.

7. H.L. Stone. Iterative solution of implicit approximations of multi-dimensional partial differential equations. SIAM J. Numer. Anal., 5:530-558, 1968.

8. H.A. van der Vorst. Iterative Krylov Methods for Large Linear systems. Cambridge University Press, Cambridge, 2003.

9. F. Vermolen, C. Vuik, and A. Segal. Deflation in preconditioned conjugate gradient methods for finite element problems. In M. Krǚžek, P. Neittaanmäki, R. Glowinski, and S. Korotov, editors, Conjugate Gradient and Finite Element Methods, pages 103-129. Springer, Berlin, 2004.

10. R.L. Verweij. Parallel Computing for furnace simulations using domain decomposition. PhD thesis, Delft University of Technology, Delft.

11. C. Vuik and J. Frank. Coarse grid acceleration of a parallel block preconditioner. Future Generation Computer Systems, 17:933-940, 2001.

12. P. Wesseling. Principles of Computational Fluid Dynamics. Springer, Heidelberg, 2001. 